DOE/ER/60565-6

\title{
COMPUTER ASSISTED MULTIPLEX SEQUENCING
}

Progress Report

for the period of August 1, 1991 to July 31, 1992

George M. Church

Department of Genetics, EQRF

Harvard Medical School

200 Longwood Avenue

Boston, MA 02115

August 1992

Prepared for

THE U.S. DEPARTMENT OF ENERGY

GRANT NUMBER: DE-FG02-87ER60565

MASTER

NOTICE:

DISTRIBUTION OF THIS DOCUMENT IS UNLIMUTED

This report was prepared as an account of work sponsored by the United States Government. Neither the United States nor the

Department of Energy, nor any of their employees, nor any of their contractors, subcontractors, or their employees, makes any warranty, express or implied, or assumes any legal liability or responsibility for the accuracy, completeness, or usefulness of any information, apparatus, product or process disclosed or represents that its use would not infringe privately-owned rights.

\section{DISCLAIMER}




\section{Abstract:}

The objectives of this project are automation and optimization of multiplex sequencing. This year we have integrated direct transfer electrophoresis, automated multiplex hybridizations and automated film reading and applied this toward sequencing of three contiguous E. coli cosmids. Primers for the directed dideoxy sequence walking and sequence confirmation steps were synthesized with a 15 base tag complimentary to an alkaline phosphatase conjugate. A higher throughput synthesis device is well along in testing as are new automated hybridization devices. We have developed software cor automatically annotating ORFs and databases of precise termini of proteis and RNA.

\section{Technical progress during the current budget period:}

The objectives of this project are automation and rptimization of multiplex sequencing. These objectives often fit in well with the needs of other non-multiplex based sequencing methods in particular gel based or hybridization based sequencing methods.

We have this year integrated direct transfer electrophoresis, automated multiplex hybridizations and automated film reading and applied this toward sequencing of three contiguous E. coli cosmids. C-specific hydrogen peroxide reactions eliminate the need for hydrazine (and hence precipitations and hazards). Sequence patterns for two cosmids were detected using chemiluminescence with oligonucleotide probes directly conjugated to alkaline phosphatase. Primers for the directed dideoxy sequence walking and sequence confirmation steps were synthesized with a 15 base tag complimentary to an alkaline phosphatase conjugate. Sequence patterns for the third cosmid were detected using radiolabled oligonucleotide probes. Film data were automatically read and assembled using the programs REPLICA and GTAC. For the cosmids, 20 gels resulted in 9216 sequences on film. (Laura Jaehn and Hamid Ghazizadeh were responsible for computer data management).

We have developed a program which automatically finds and graphically annotates ORFs including matches to database sequences. Among many other features in the cosmids, this turned up new potential operon(s) covering 13 consecutive long ORFs without homologs in any databases. This is by far the longest found in E.coli or Salmonella to our knowledge. These programs will allow us to 
submit these cosmid sequences to Genbank within a week of the final sequence confirmation pass which we expect in September.

Minimal annotation for sequence database contributions typically includes inferred homologies and gene products. To help aid programs making such inferences we have established a database of the 400 most abundant E.coli proteins $\mathrm{N}$-terminal sequences, pI, MW, abundance, and subcellular localization. A draft version of this has been sent to Ken Rudd at NCBI and Fred Blattner at U. Wisconsin. In addition, to be maximally useful as a reference protein sequence collection the E.coli genomic database is expected to have few or no completely uncharacterized ORFs. The above protein database was established by Andy Link in our laboratory using sub-cellular and 2D gel fractionations plus automated sequencing to quantitate and establish the $12 \mathrm{~N}$-terminal residues. Blast searches were generally unambiguous hits, ORFs, or unknowns. These studies are being extended by the establishment of combined multiplex sequencing and gene mutation/replacement vectors compatible with many strains (including wild types) of enterics. Whole genome libraries have been constructed. Alec Mian and Richard Chin are working on extending the concept of systematic study of protein termini to that of RNA termini and regulatory sites. Such a database is vital for testing algorithms for prediction of sites in genomic sequences. In order to avoid ambiguities in the identity of terminal bases Alec Mian has established a oligomer attachment procedure based on RNA ligase and PCR and has used this procedure to sequence several nonpolyadenylated transcripts from repetitive regions.

In order to increase the number of oligonucleotides synthesized in parallel and decrease the scale of synthesis, we have explored the automation of multiplex solid phase DNA synthesized. Synthesis occurs on the surface of pins which are dipped into appropriate common troughs containing reagent, including modified monomers. The pins are controlled via a solenoid array set on a 96 -well $9 \mathrm{~mm}$ spacing and the troughs are positioned via a stepping motor. Yields are typically in the 100 picomole range. The products are assayed by priming DNA sequencing, PCR, kinase labeling and gel electrophoresis. By these assays synthetic oligonucleotides made are comparable in quality to those made on commercially available synthesizers. Versions of the machine capable of the simultaneous synthesis of 4,8 , and 20 different oligomers have been developed and tested. This work was done by George Church and Jim Horn in 
our machine shop (under the primary project responsibility of Steve Kieffer-Higgins who is supported by HHMI).

Working with Bruce Nappi at Foster-Miller, Inc. we have developed new automated hybridization/exposure devices (their end supported by an NIH SBIR grant). The first device from this project will be tested in our laboratory at the end of this month. This design has membranes on the outside of drum. Previous devices made in our lab have had membranes on the inside of drums (used for 3 cosmids see above), inside of rectangular tanks, and inside of flowinjected thin laminated bags.

Working with Jen-i Mao and Doug Smith at nearby Collaborative Research Inc., we have set up a complete industrial-scale multiplex sequencing system including all of our hardware and software for use in sequencing Mycobacterial genomes. In their first 10 months (in addition to hiring, training, setting up, etc.) they have sequenced 4 cosmids and are well along on 4 more. Their first year production could easily be better than most the 3 year efforts of other largescale sequencing laboratories. We are very encouraged by this, and plan to continue this collaboration wherein they can incorporate our tested modifications in a production setting and help to determine the exact degree of enhancement of accuracy and/or speed.

Proposed research for the coming year if different from the original proposal: The research is continuing essentially as originally proposed.

\section{Recent and ongoing technology transfer:}

(These are the same as last year except addition of the first item)

Foster-Miller, Inc. Development of new automated hybridization/exposure device designs.

Betagen Inc., Polytech Products and others Direct transfer electrophoresis device improvements. 
BioComp Inc. An automated hybridization and chemiluminescent development system for uniplex or multiplex sequencing.

Collaborative Research Inc. Complete multiplex sequencing including all hardware and software for use in sequencing Mycobacterial genomes.

Hamilton Inc. Precision 12 channel gel loader for sequencing gels and agarose gels using standard 96 well spacing. These are compatible with" gels slots as small as 80 microns by $2.25 \mathrm{~mm}$.

Milligen/Biosearch/Millipore Inc. Multiplex DNA sequencing system, vectors, primers, devices for colony picking and automated probing, alkaline phosphatase oligonucleotide conjugates for non-radioactive DNA sequencing, UV crosslinking of nucleic acids to nylon membranes.

New England Biolabs Inc. Collaborators on the multiplex system above.

Owl Scientific Inc. Sequencing sharkstooth combs based on 96 well spacing (integral multiples: $4.5,3.0,2.25 \mathrm{~mm}$ ).

TeraBase Inc. A multipin automated oligonucleotide chemical synthesis device.

Tropix Inc. Electrotransfer of sequencing gels for non-radioactive DNA sequencing detection. 

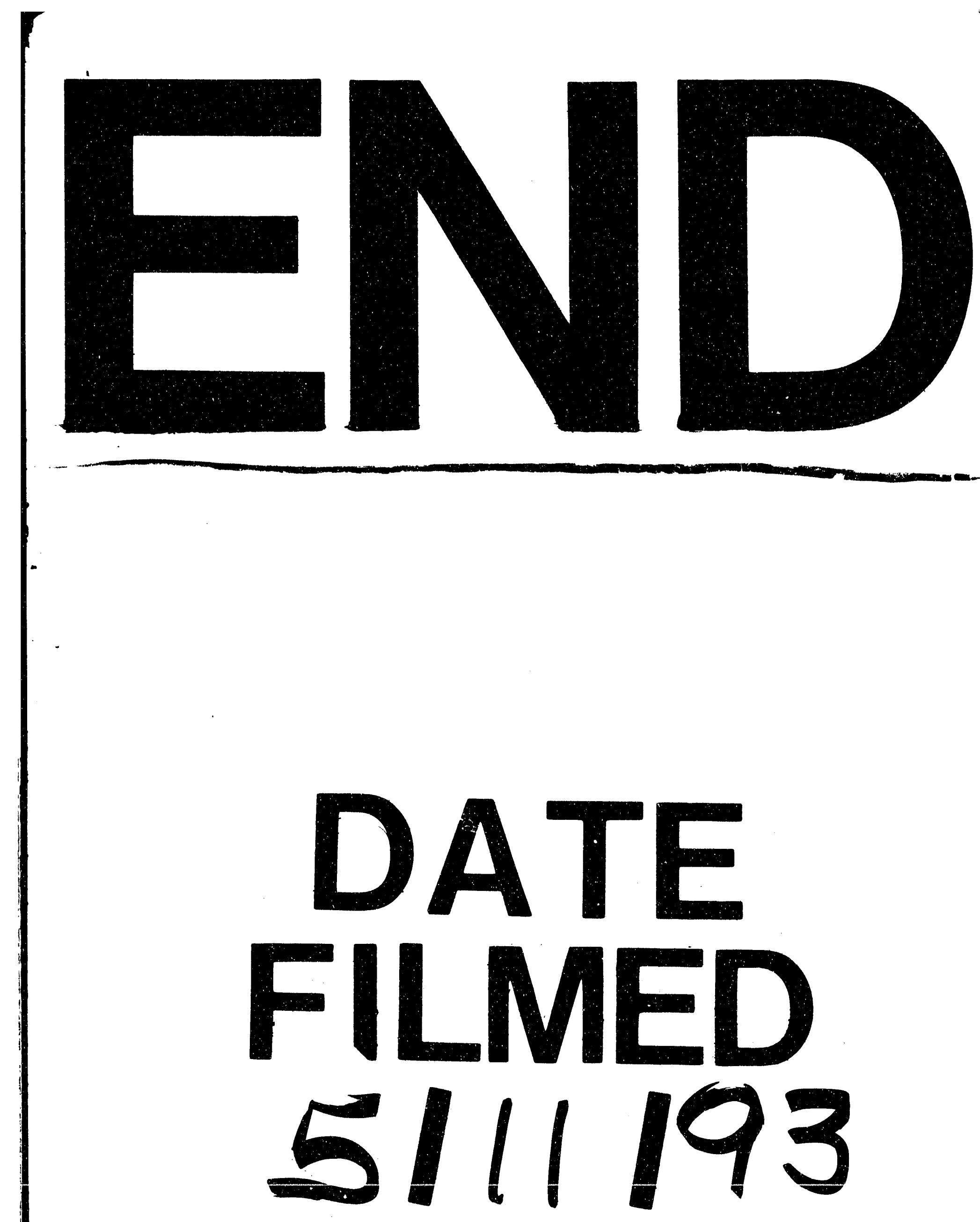
